\title{
Optical Characterization of DebriSat Fragments in Support of Orbital Debris Environmental Models
}

\author{
Heather M. Cowardin ${ }^{1} \cdot$ John M. Hostetler ${ }^{2}$ - James I. Murray ${ }^{2}$. \\ Jacqueline A. Reyes ${ }^{3}$. Corbin L. Cruz ${ }^{2}$
}

Accepted: 8 July 2021/ Published online: 27 October 2021

This is a U.S. government work and not under copyright protection in the U.S.; foreign copyright protection may apply 2021

\begin{abstract}
The NASA Orbital Debris Program Office (ODPO) develops, maintains, and updates orbital debris environmental models, such as the NASA Orbital Debris Engineering Model (ORDEM), to support satellite designers and operators by estimating the risk from orbital debris impacts on their vehicles in orbit. Updates to ORDEM utilize the most recent validated datasets from radar, optical, and in situ sources to provide estimates of the debris flux as a function of size, material density, impact speed, and direction along a mission orbit. On-going efforts within the NASA ODPO to update the next version of ORDEM include a new parameter that highly affects the damage risk - shape. Shape can be binned by material density and size to better understand the damage assessments on spacecraft. The in situ and laboratory research activities at the NASA ODPO are focused on cataloging and characterizing fragments from a laboratory hypervelocity-impact test using a high-fidelity, mockup satellite, DebriSat, in controlled and instrumented laboratory conditions. DebriSat is representative of present-day, low Earth orbit satellites, having been constructed with modern spacecraft materials and techniques. The DebriSat fragment ensemble provides a variety of shapes, bulk densities, and dimensions. Fragments down to $2 \mathrm{~mm}$ in size are being characterized by their physical and derived properties. A subset of fragments is being
\end{abstract}

This article belongs to the Topical Collection: Advanced Maui Optical and Space Surveillance Technologies (AMOS 2020)

Guest Editors: James M. Frith, Lauchie Scott, Islam Hussein

An earlier version of this article was first presented at the 21st Advanced Maui Optical and Space Surveillance Technologies Conference, held in Wailea, Maui, Hawaii, September 15-18, 2020.

Heather M. Cowardin

heather.cowardin@nasa.gov

1 NASA Johnson Space Center, Orbital Debris Program Office, 2101 NASA Pkwy, Houston, TX 77058, USA

2 Jacobs JETS Contract, NASA Johnson Space Center, 2101 NASA Pkwy, Houston, TX 77058, USA

3 University of Texas at El Paso, 500 W. University Ave, El Paso, TX 79968, USA

를 Springer 
analyzed further in NASA's Optical Measurement Center (OMC) using broadband, bidirectional reflectance measurements to provide insight into the optical-based NASA Size Estimation Model. Additionally, pre-impact spectral measurements on a subset of DebriSat materials were acquired for baseline material characterization. This paper provides an overview of DebriSat, the status of the project, and ongoing fragment characterization efforts within the OMC.

Keywords orbital debris $\cdot$ laboratory $\cdot$ hypervelocity impact test $\cdot$ environmental models

\section{Introduction}

The DebriSat project is a collaboration of the NASA Orbital Debris Program Office (ODPO), the Air Force Space and Missile Systems Center (SMC), The Aerospace Corporation (Aerospace), the University of Florida (UF), and the Air Force Arnold Engineering Development Complex (AEDC). The project has four primary goals: 1) design and fabricate a 56-kg class spacecraft ("DebriSat") representative of modern spacecraft in the low Earth orbit (LEO) environment; 2) conduct a hypervelocity laboratory impact test to simulate a catastrophic fragmentation event of DebriSat; 3) collect, measure, and characterize all fragments down to $2 \mathrm{~mm}$ in size; and 4) use the data to improve space situational awareness applications and satellite breakup models for better orbital debris environment definition [1].

The motivation for the DebriSat project was based on a key impact test series, Satellite Orbital Debris Characterization Impact Test (SOCIT), which was conducted by the Department of Defense (DOD) and NASA at AEDC in 1992 to support the development of satellite breakup models. The main target for SOCIT was a fully functional U.S. Navy Transit 1960's era satellite. The DOD and NASA breakup models based on the SOCIT data have supported many applications and matched on-orbit events reasonably well over the years [1].

New laboratory-based tests to acquire data to improve the existing DOD and NASA breakup models are needed as new materials and construction techniques are developed for modern satellites. The need for such tests is supported also by discrepancies between model predictions and observations of fragments generated from the breakup of modern satellites, including the Iridium 33 and Fengyun 1-C [13].

The DebriSat design was based on a survey of modern satellites in LEO, as discussed in two 2013 University of Florida dissertations: M. Werremeyer's "Design of Sub-systems for a Representative Modern LEO Satellite" and S. Clark's "Design of a Representative LEO Satellite and Hypervelocity Impact Test to Improve the NASA Standard Breakup Model." All major design decisions, including the selection of components, subsystems, mass fractions, structure, and construction methods, were reviewed and approved by Aerospace subject matter experts. In addition, the DebriSat body was covered with multi-layer insulation (MLI) and three solar panels were attached to one side of the main body (Fig. 1).

To reduce the project cost, a decision was made to emulate the majority of components. The emulated components were based on existing designs of flight hardware, including structure, dimensions, materials, and connection mechanisms. At the end of the assembly, DebriSat was subjected to a NASA General Environmental Verification Specification qualification vibration test to ensure the integrity of the structure.

To increase the project's benefits further, Aerospace designed and built a target resembling a launch vehicle upper stage ("DebrisLV") for the pre-test shot. The pre-test 

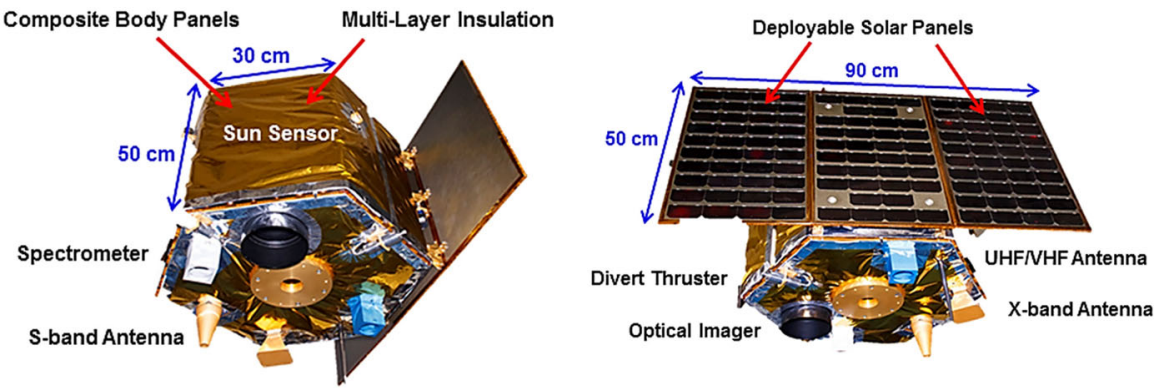

a)

b)

Fig. 1 Illustration of the DebriSat satellite from two different perspectives. View a) shows hexagonal satellite with the composite body panels each measuring $50 \mathrm{~cm} \times 30 \mathrm{~cm}$. The body of the satellite is wrapped in multilayered insulation. View b) shows the satellite with the $50 \mathrm{~cm} \times 90 \mathrm{~cm}$ deployable solar panels. In both views various components are identified including the sun sensor, spectrometer, S-band antenna, divert thruster, optical imager, UHF/VHF antenna, and x-band antenna (Credit: University of Florida)

shot was conducted two weeks prior to the DebriSat impact test with identical conditions to DebriSat (facility set-up, projectile, and impact speed). The resulting fragments were collected in boxes and will be characterized after DebriSat characterization is complete. Fig. 2 shows the mounting of DebrisLV inside the target chamber.

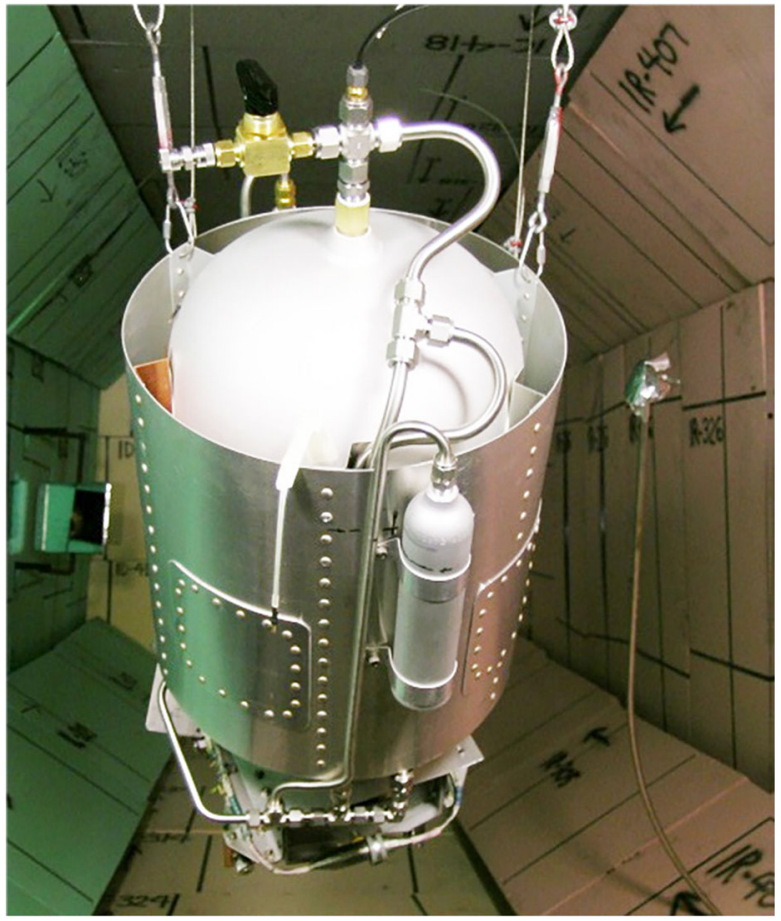

Fig. 2 Digital image of DebrisLV mounted inside the impact chamber at AEDC. DebrisLV was used as a pretest shot conducted two-weeks prior to the DebriSat test and built to resemble a vehicle upper stage, named "DebrisLV." The cylindrical target is composed of various metals and is shown hung inside the foam enclosed chamber prior to impact (Credit: Arnold Engineering Development Complex/Air Force) 
On 1 April and 15 April 2014, respectively, the DebrisLV and DebriSat impacts were successfully carried out at AEDC Range G. To maximize the projectile mass at the $7 \mathrm{~km} / \mathrm{sec}$ impact speed without a sabot, the AEDC team developed a special projectile design featuring a hollow aluminum cylinder embedded in a nylon cap. The nylon cap served as a bore rider for the aluminum cylinder to prevent hydrogen leakage and to protect the two-stage light gas gun barrel [14]. Table 1 shows a comparison of some SOCIT, DebriSat, and DebrisLV test conditions. After the impacts of DebrisLV and DebriSat, all soft catch panels used inside the chamber to minimize secondary damage, loose fragments, and dust were carefully collected, processed, documented, and placed in bags or plastic containers for shipping to a storage facility. The initial estimates using the NASA Standard Satellite Breakup Model (SSBM) indicated the number of $2 \mathrm{~mm}$ (and larger) fragments from DebriSat and DebrisLV were approximately 85,000 and 35,000, respectively. Since August 2014, the UF team has been working to extract fragments from foam panels, characterize each fragment down to $2 \mathrm{~mm}$ in size, and upload the data into a database. Figure 3 shows the number of fragments recorded in the database and the estimated numbers of fragments collected to be analyzed in comparison to the SSBM prediction since the year the experiment was conducted through fiscal year (FY) 2019. Note, the need to update the SSBM is evident utilizing laboratory break-up experiments that encompass more modern construction methods and materials, as well as classical represented satellites, such as SOCIT, to improve breakup models used by NASA and DOD.

\section{DebriSat Characterization \& Analysis}

All fragments with at least one dimension $\geq 2 \mathrm{~mm}$ are carefully collected and/or extracted from the foam panels/pieces and assigned unique identification numbers before their physical characteristics are determined. During the characterization process, each

Table 1 Experimental details on SOCIT, DebriSat, and DebrisLV test campaigns

\begin{tabular}{|c|c|c|c|}
\hline & SOCIT/ Transit & DebriSat & DebrisLV \\
\hline $\begin{array}{l}\text { Target body } \\
\text { dimensions }\end{array}$ & $46 \mathrm{~cm}(\mathrm{dia}) \times 30 \mathrm{~cm}(\mathrm{ht})$ & $60 \mathrm{~cm}(\mathrm{dia}) \times 50 \mathrm{~cm}(\mathrm{ht})$ & $35 \mathrm{~cm}($ dia $) \times 88 \mathrm{~cm}(\mathrm{ht})$ \\
\hline Target mass & $34.5 \mathrm{~kg}$ & $56 \mathrm{~kg}$ & $17.1 \mathrm{~kg}$ \\
\hline MLI and solar panel & No & Yes & No \\
\hline Projectile material & Al sphere & $\begin{array}{l}\text { Hollow Al cylinder with } \\
\text { attached nylon bore-rider }\end{array}$ & $\begin{array}{l}\text { Hollow Al cylinder with } \\
\text { attached nylon bore-rider }\end{array}$ \\
\hline $\begin{array}{l}\text { Projectile } \\
\text { dimension/mass }\end{array}$ & $4.7 \mathrm{~cm}$ diameter, $150 \mathrm{~g}$ & $8.6 \mathrm{~cm} \times 9 \mathrm{~cm}, 570 \mathrm{~g}$ & $8.6 \mathrm{~cm} \times 9 \mathrm{~cm}, 598 \mathrm{~g}$ \\
\hline Impact speed & $6.1 \mathrm{~km} / \mathrm{s}$ & $6.8 \mathrm{~km} / \mathrm{s}$ & $6.9 \mathrm{~km} / \mathrm{s}$ \\
\hline $\begin{array}{l}\text { Impact Energy to } \\
\text { Target Mass ratio } \\
\text { (EMR) }\end{array}$ & $81 \mathrm{~J} / \mathrm{g}(2.8 \mathrm{MJ}$ total $)$ & $235 \mathrm{~J} / \mathrm{g}(13.2 \mathrm{MJ}$ total $)$ & $832 \mathrm{~J} / \mathrm{g}(14.2 \mathrm{MJ}$ total $)$ \\
\hline $\begin{array}{l}\text { Soft-Catch System: } \\
\text { Polyurethane } \\
\text { foam stacks }\end{array}$ & $\begin{array}{l}3 \text { densities: } 0.06,0.096 \\
\text { and } 0.192 \mathrm{~g} / \mathrm{cm}^{3} \\
25 \mathrm{~cm} \text { thick }\end{array}$ & $\begin{array}{l}3 \text { densities: } 0.048,0.096 \text {, } \\
\text { and } 0.192 \mathrm{~g} / \mathrm{cm}^{3} \text {; up to } \\
61 \mathrm{~cm} \text { thick }\end{array}$ & $\begin{array}{l}3 \text { densities: } 0.048,0.096 \text {, } \\
\text { and } 0.192 \mathrm{~g} / \mathrm{cm}^{3} \text {; up to } \\
51 \mathrm{~cm} \text { thick }\end{array}$ \\
\hline
\end{tabular}




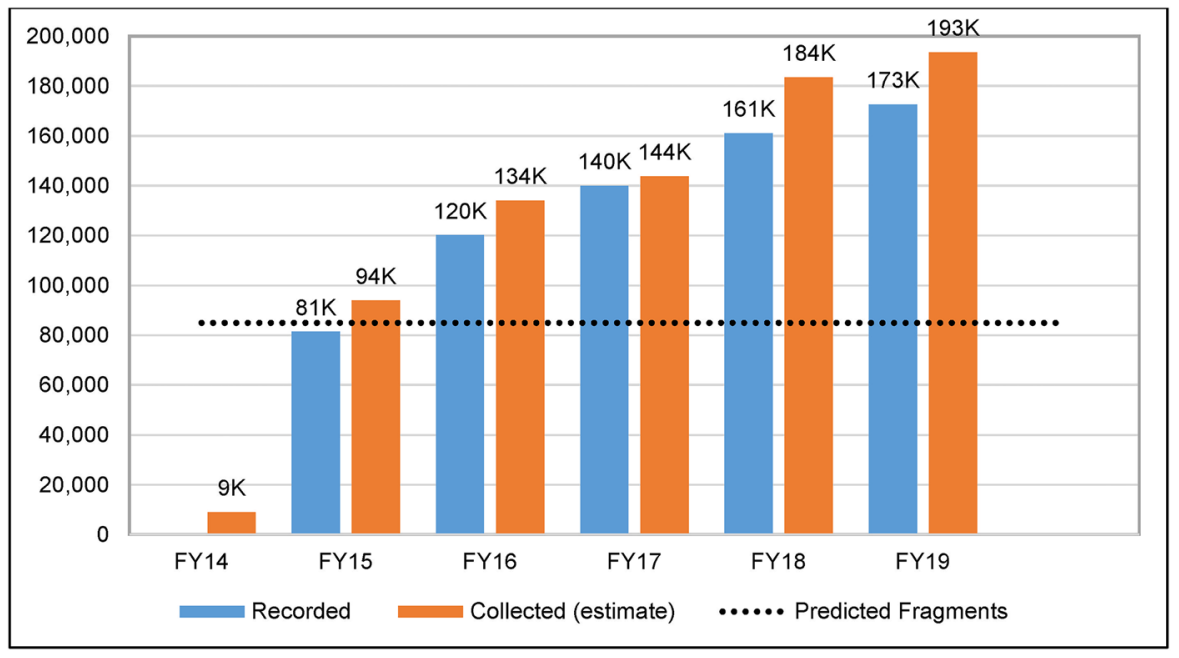

Fig. 3 This figure shows a two data set bar plot of the number of recorded fragments in the database and the estimated number of collected fragments from FY14 through FY19. The initial estimate for number of fragments was calculated to be 85,000 , but that value was superseded shortly after one year of fragment characterization. At the end of FY19, the project had collected approximately 193,483 fragments and recorded 172, 625 fragments (Credit: University of Florida)

fragment's physical (observed and derived) parameters are archived in the DebriSat Categorization System (DCS). The DCS is a database solution designed and developed specifically to manage the large amounts of data generated by the DebriSat project. In addition to the information shown in Table 2, associated metadata (e.g., location the fragment was found within the chamber, images of the fragments, etc.) are also archived in the DCS. The initial step involves assessing each uniquely identified fragment in terms of material, shape, and color. Mass measurements are acquired using a suite of scales appropriate for the individual fragment's mass. To optimize the characterization process and implement methodologies to minimize biases and/or errors associated with "human-inthe-loop" activities during measurements, 2D and 3D imagers are utilized to measure the physical size based on the solid body (SB) dimensions (XSB, YSB, and ZSB). From these initial measurements, the characteristic length $\left(\mathrm{L}_{\mathrm{c}}\right)$, the average of the object's three maximum orthogonal projected dimensions (XDIM, YDIM, and ZDIM), average crosssectional area, volume, and bulk density are calculated and stored in the DCS. The $\mathrm{L}_{\mathrm{c}}$ is a common parameter used to represent size as it can be translated from observable parameters, such as radar cross section (RCS) via the NASA Size Estimation Model (SEM). This geometrical mean accounts for removing any aspect angle dependencies during observations and provides an averaged size dimension for modeling purposes. Note, the initial set of fragments investigated concentrated on carbon fiber reinforced polymers (CFRP) using the 2D imagers where the third dimension (i.e., ZDIM) could be extracted from the material's density. As the project has progressed from these initial homogenous fragments, mirrors have been implemented in the 2D imaging system to calculate the third dimension. More details on the characterization can be found in $[5,17]$ (Table 2).

The current NASA SSBM was formulated using laboratory tests and ground-based remote measurements of on-orbit fragmentation events to provide an average breakup ensemble for spacecraft and upper stage collisions and explosions. Significant 
Table 2 Fragment Characterization Parameters stored in the Debris Categorization System

\begin{tabular}{|c|c|}
\hline Parameter & Definition/Notes \\
\hline $\begin{array}{l}\text { Unique ID with associated } \\
\text { barcode }\end{array}$ & $\begin{array}{l}\text { Earlier studies grouped multiple, similar small fragments to get average } \\
\text { characteristics }\end{array}$ \\
\hline Material & $\begin{array}{l}\text { Predefined categories based on as-built design; material density is auto-populated } \\
\text { once material is selected. }\end{array}$ \\
\hline Shape & $\begin{array}{l}\text { Flat Plate } \\
\text { Bent Plate } \\
\text { Straight Needle/Rod/Cylinder } \\
\text { Bent Needle/Rod/Cylinder } \\
\text { Parallelepiped/Nugget/Spheroid } \\
\text { Flexible/MLI }\end{array}$ \\
\hline Color & Predefined categories based on as-built design \\
\hline Principle dimensions & $\mathrm{x}, \mathrm{y}, \mathrm{z}(\mathrm{mm})\{$ relative to solid body coordinates \\
\hline Characteristic length & $L_{c}=\left(X_{-} D I M+Y_{-} D I M+Z_{-} D I M\right) / 3(\mathrm{~mm})$ \\
\hline $\begin{array}{l}\text { Average cross-sectional } \\
\text { area }\end{array}$ & $\begin{array}{l}\text { Weighted average of projected areas visible in multiple } 2 \text {-D images; } \\
\text { pixel-to-length (and area) relationship calculated from hardware characteristics } \\
\text { and scene geometry }\left(\mathrm{mm}^{2}\right) \text {. }\end{array}$ \\
\hline Mass & Fragment mass $(\mathrm{g})$ \\
\hline Area-to-mass ratio (AMR) & Calculated average cross-sectional area divided by measured mass $\left(\mathrm{mm}^{2} / \mathrm{g}\right)$. \\
\hline Volume & $\begin{array}{l}\text { Calculated from point cloud using convex hull and alpha-shape subtraction } \\
\left(\mathrm{mm}^{3}\right) \text {. }\end{array}$ \\
\hline Bulk Density & Measured mass divided by calculated volume $\left(\mathrm{g} / \mathrm{mm}^{3}\right)$. \\
\hline
\end{tabular}

validation efforts against observable data were conducted, including the development of a radar Size Estimation Model (SEM) based on SOCIT fragments. To assess how to best update or improve future environmental models, such as the Orbital Debris Engineering Model (ORDEM) and the SSBM, a statistical assessment must be done to determine the distribution of key parameters (shape, density, and size) using laboratory data.

The SSBM uses $\mathrm{L}_{c}$ as the fundamental independent variable in lieu of mass. This choice of $\mathrm{L}_{\mathrm{c}}$ in preference to mass was driven by the on-orbit observables, namely Two-Line Element (TLE) orbital data sets and RCS time series. More details on the SSBM can be found in [5, 17]. In Fig. 4 the cumulative number as a function of $L_{c}$ is shown binned by primary material type. Due to the project focus on CFRP during the initial DebriSat fragment analysis, the number of CFRP fragments may be biased in the overall material assessment. Fig. 5 shows cumulative number as a function of mass, also binned by material type. Currently, CFRP dominates the small fragment material type in both size and mass categories, but as these parameters increase the metals dominate the population. As the characterization process continues, the trend assessment is subject to change. The transition from predominantly CFRP to metal is currently around $2 \mathrm{~cm}$ in $\mathrm{L}_{\mathrm{c}}$ (see Fig. 4) and 10 milligrams (see Fig. 5).

Fig. 6 shows the cumulative number distribution of fragments as a function of $\mathrm{L}_{\mathrm{c}}$ categorized by shape categories. Below $\mathrm{L}_{c}$ of approximately $20 \mathrm{~mm}$, the CFRP dominate the material category, which happen to be mostly flat plates and "needlelike" straight rod fragments. As the distribution increases in size, the larger fragments 


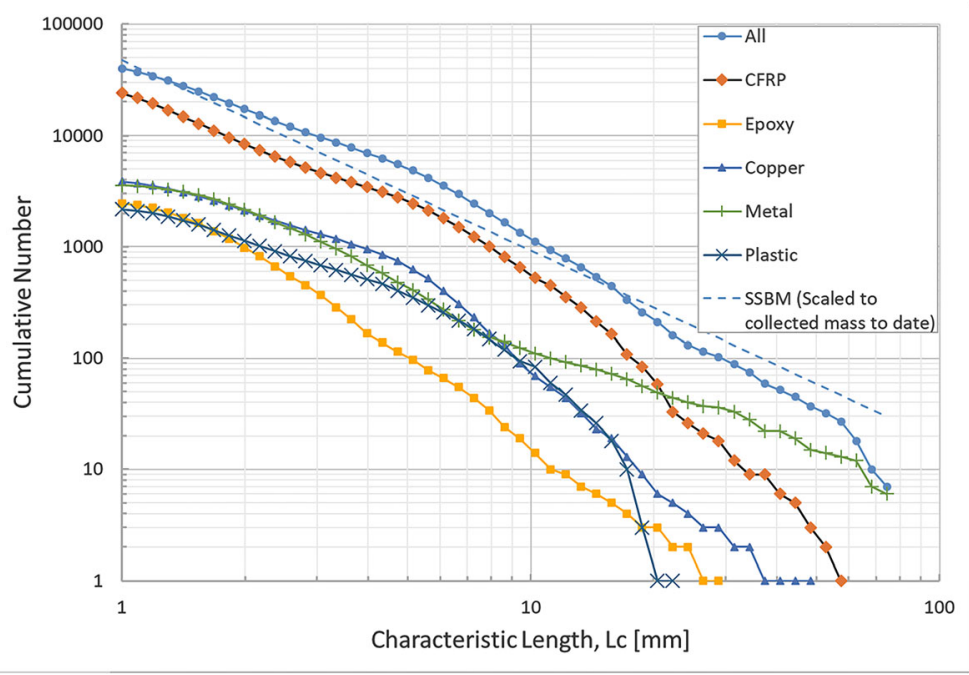

Fig. 4 Cumulative number of fragments versus characteristics length $\left(\mathrm{L}_{\mathrm{c}}\right)$ as a function of material as of 16 January 2020. The materials include CFRP, Epoxy, Copper, Metal, and Plastic. The materials are all scaled by collected mass to date to NASA's Standard Satellite Breakup Model (SSBM). The figure shows CFRP dominates the smaller size regime and metal transition is prevailing after approximately $2 \mathrm{~cm}$

are classified as parallelepiped/nugget/spheroid. As the fragments continue to be analysed, these classifications will continue to evolve. Further distributions by density and AMR can be viewed in Ref. [17].

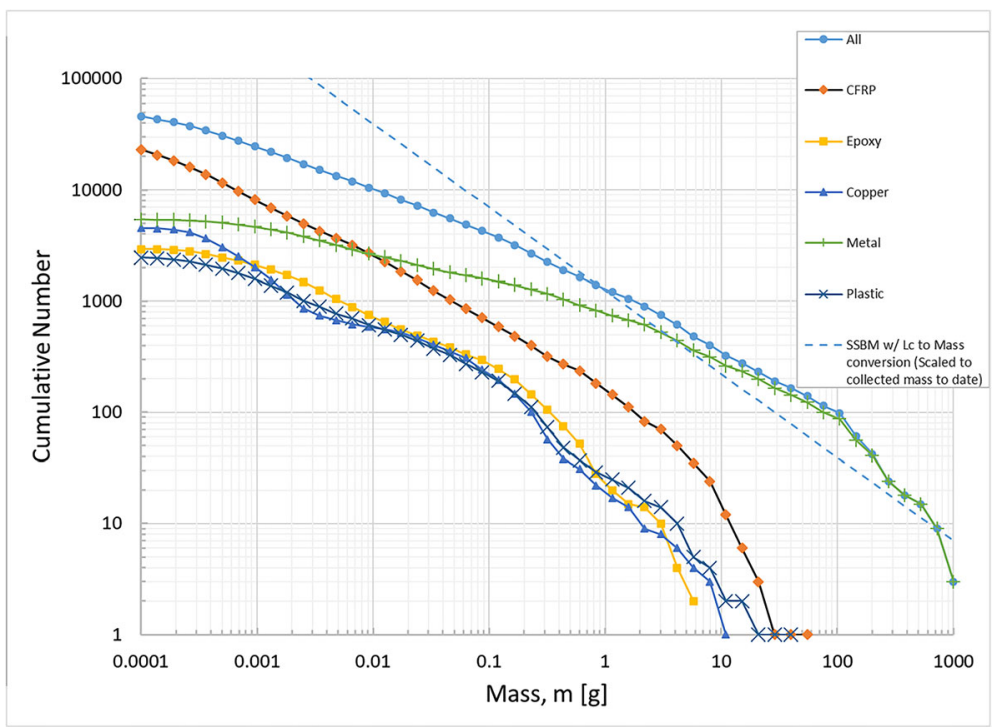

Fig. 5 Cumulative number of fragments versus mass as a function of material as of 16 January 2020. Like Fig. 5, the materials include CFRP, Epoxy, Copper, Metal, and Plastic. The materials are also scaled by collected mass to date to NASA's Standard Satellite Breakup Model (SSBM) but uses the characteristic length to mass conversion. The figure shows CFRP dominates the smaller mass regime and the transition to metal outweighing other materials occurs at approximately $10 \mathrm{mg}$ 


\section{Optical Characterization}

The NASA ODPO has utilized optical observations of Earth-orbiting objects to better characterize the orbital debris environment from low Earth orbit, to geosynchronous orbit, and to geostationary transfer orbit. Ground-based measurements provide time-dependent orbital parameters and brightness (i.e., magnitudes). The magnitude data is converted into size assuming a range, phase function, and albedo. To better model the optical size to target size, as RCS is equated to $\mathrm{L}_{\mathrm{c}}$ using the SEM size conversation as a function of radar wavelength, data acquired in the OMC allow optical data products to be measured using space-based illumination conditions, equipment, and techniques that parallel telescopic observations and source-target-sensor orientations (Fig. 7). To improve upon the current optical size estimation, the $\mathrm{OMC}$ is investigating new phase functions based on spacecraft material samples and fragments from laboratory hypervelocity impact tests, such as DebriSat, with plans to generate a distribution of phase functions.

\section{Photometric Measurements/BRDF}

To improve the sampled brightness of laboratory targets, the aspect-angle dependencies in object measurements are removed by fixing the object to the end effector of a six degree-offreedom robotic arm and sampling a hemisphere of object view orientations. The totality of this sampled hemisphere is analogous to the bidirectional reflectance distribution function (BRDF), defined by Nicodemus, et al. [18], as a radiometric concept that identifies the reflective characteristics of a surface by the ratio of reflected radiance in the direction $\left({ }_{r}, \psi_{\mathrm{r}}\right)$ to the radiation flux incident on a surface from direction $\left({ }_{i}, \psi_{i}\right)$ within the solid angle element $\mathrm{d} \omega_{\mathrm{i}}[10]$. In the OMC, a BRDF is constructed as a hemispheric surface interpolated from laboratory measurements made at 21 discrete view orientations (Fig. 8). Intensity curves (i.e.,

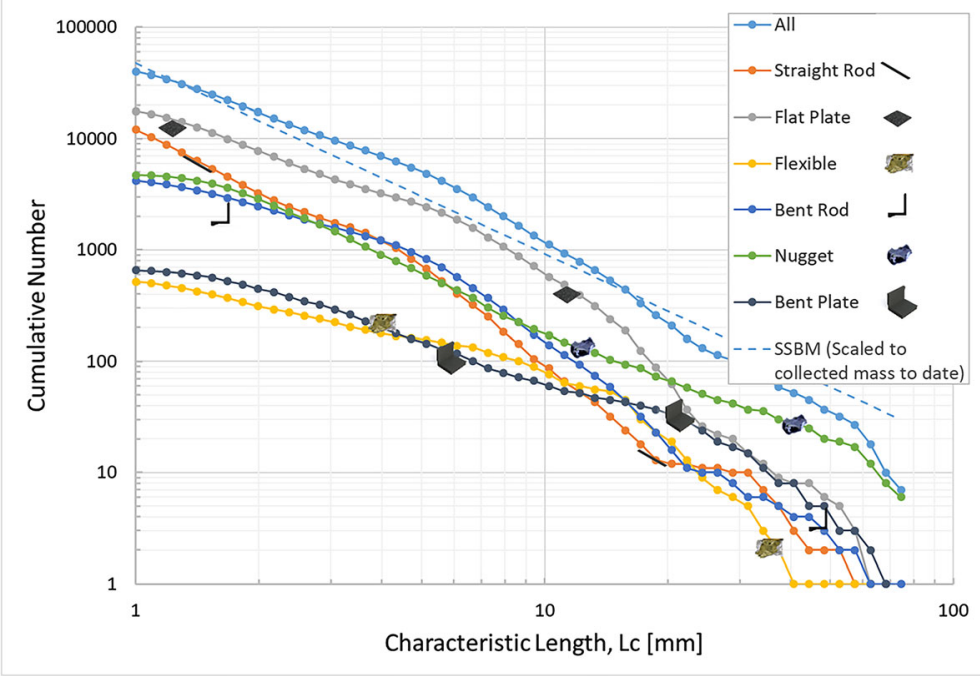

Fig. 6 Cumulative number of fragments versus characteristics length $\left(\mathrm{L}_{\mathrm{c}}\right)$ as a function of six shape categories as of 16 January 2020. For objects less than $3 \mathrm{~mm}$, the flat plates represent the majority of fragments, followed by straight rods, nuggets, bent rods, bent plates, and flexible shapes, in that order. For fragments larger than $2 \mathrm{~cm}$ the nuggets dominate the shape category, with the remaining shapes following with low count numbers 


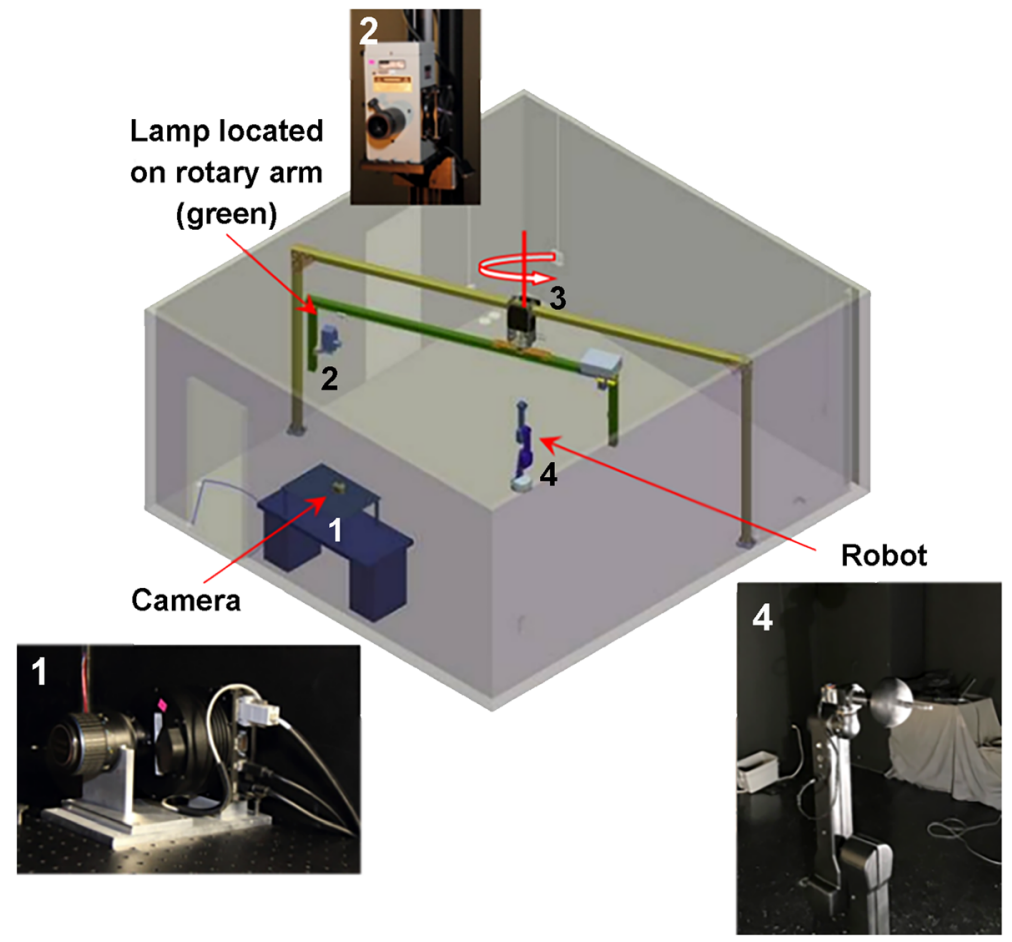

Fig. 7 Graphic showing the Optical Measurement Center (OMC) located at NASA JSC. The OMC consists of five primary instruments/components: 1) CCD camera (shown positioned on optical bench), 2) Xenon arc lamp (shown mounted on rotary arm), 3) rotary arm secured to steel structure that allows full 360 degree sweep in facility, 4) six degree-of-freedom robotic arm cantered in facility for positioning fragments, and 5) ASD Field Spectrometer (not pictured in image)

linear dataset) represent instrumental data products, whereas magnitude is normalized to a Lambertian calibration target to compute absolute magnitudes for each target's reflectance distribution. By normalizing the BRDF to that of a Lambertian sphere, the BRDF represents all possible values of the object's phase function at each phase angle in the solar range.

Previous work studied the relation between an object's surface optical properties on subsequent behaviors exhibited in their phase functions. By imaging several objects with identical sizes and shapes but varying optical properties, it was discovered that the dominance of diffuse versus specular surfaces resulted in unique characteristics in their associated phase function. These preliminary findings suggest that it may be possible to discern an object's material composition from these photometric functions. With the material composition confirmed, the overall geometric albedo, $\alpha_{g}$, may be subsequently approximated, and the phase function $f(\phi)$ may also be directly constructed using photometric data collected when sampling the BRDF [11]. Therefore, the OMC intends to image a multitude of fragments generated via high velocity impact testing and cataloging their experimentally derived BRDFs. By imaging these objects, which are representative of objects present in the orbital debris environment, a more general understanding of an object's material composition impact on phase function behavior is sought, with the ultimate goal of providing a means for identifying these characteristics for data collected via telescopic measurements. 

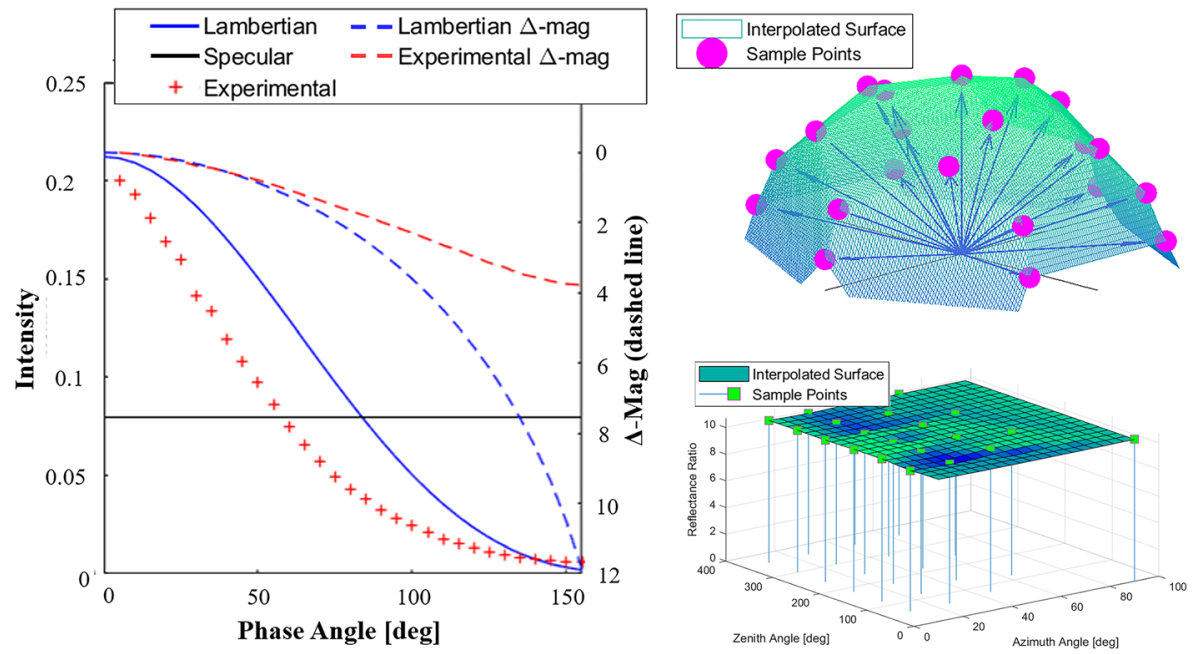

Fig. 8 Three plots are shown in this image, starting on the left, with an intensity versus phase angle plot for a Lambertian sphere target from $\sim 5$-degree phase angle to 155 -degree phase angle. The Lambertian phase angle (linear and delta-magnitude) is represented by solid and dashed blue lines, Experimentally derived-phase function (linear and delta-magnitude) by cross-hatched and dashed red lines, and Specular represented by a solid black line. Second, top-right, shows a hemispherical plot with 21 discrete sampling points connected via an interpolated surface. Last, bottom- right, a 3-D plot showing the sample points discussed above, as a function of Zenith angle (degrees), Azimuth angle (degrees) and apparent magnitude [11]

\section{Phase Function Investigation}

The accepted model for approximating target size from calibrated magnitudes is the optical-based SEM developed by NASA's Orbital Debris Program Office, which is a photometric determination utilizing both observable and assumed properties of the target. While many of the quantities utilized in the SEM are readily accessible via photometric analysis (e.g., range, phase angle, etc.), the quantities $\alpha_{g}$ (geometric albedo) and $f(\phi)$ (phase function) are largely determined by the target's shape and material composition. These properties cannot be derived solely from telescopic optical measurements, necessitating that assumptions be made to overall optical data products to derive size estimates. Conventionally, a geometric albedo of 0.175 is assumed for all objects, a value found to minimize the fractional error in resulting size estimations [2, 16], and the phase function of orbital debris is assumed to be either Lambertian or specular, although it is well understood that for rocket bodies, spacecraft, and complex shapes the phase function is a complex combination of specular and Lambertian phase functions. The geometric albedo is a byproduct of the bond albedo (ratio of incident to scattered power incorporating all wavelengths), where the bond albedo is defined as the product of the geometrical albedo of the surface and the phase function, which defines how the sunlight is scattered by the surface in the direction facing the observer. Of note, the investigation into why the global geometric albedo of 0.175 is used was based on a study of observational data products focused on fragmentation debris acquired from the Liquid Mirror Telescope with an applied Lambertian phase function correction, as well as, SEM-corrected RCS values used to calculate the global albedo value [16]. Several investigations have been initiated to supplement these assumptions in an effort to 
improve the accuracy of size estimates. Hejduk, et al., have conducted investigations into correlating material composition with phase function characteristics [9], and the OMC has also sought to provide an experimental approach for estimating an object's phase function $[10,11]$.

The objective of the OMC's phase function investigation was to replace the Lambertian assumption with an experimentally derived phase function approximation generated via imaging. After initially validating the experimental phase function process with a Lambertian sphere, four objects that were representative of orbital debris with varying optical surface properties were imaged. These objects included a small section of CFRP, an intact section of MLI, a telescope frame-cylindrical object resulting from the DebriSat experiment, and a fragment from SOCIT. Imagery and corresponding data results from the telescope frame are shown in Fig. 9 and Table 3. The experimentally determined phase functions resulted in the most accurate size estimations for the CFRP and MLI, and the shapes of their phase functions demonstrated how certain aspects of the object's geometry may be inferred. Using the known size and experimentally determined phase function, the geometric albedo for each object was determined, and this value for $\alpha_{\mathrm{g}}$ was then implemented in a second specular/Lambertian phase function size estimation. The experimentally determined values for geometric albedo were much larger than the assumed value of 0.175 , likely inflated, in part, by the short distance between the object and the CCD (i.e., range), which created a much larger solid angle subtending the target. The comparative errors using both the assumed and experimentally determined values for $\alpha_{\mathrm{g}}$ indicate that the experimental phase function is more sensitive to the geometric albedo than its specular and Lambertian counterparts. Provided these data and results, to date, the path forward will utilize OMC measurements to assess a broader, more statistically relevant sample size that represents orbital debris of various materials, geometries, and sizes. The goal is to utilize the phase function investigations and telescopic data to ascertain appropriate updates and improvements to the current optical SEM. The complexities involved with assessing a "one size fits all" geometric albedo are discussed further in the Section 3.3 Spectral Measurements (Fig. 9).

Further formulations exist which include the assumed quantities incorporated into the SEM, which may prove useful to supplement experimentally derived phase function approximations. The space illuminance ratio [9], for instance, may provide an avenue for further improving phase function approximations by understanding the impact of an object's shape on its phase function characteristics. This formulation includes both the phase function and a constant, $k$, which is specific to the observed shape, thereby providing an avenue of investigation into this relatively under-studied aspect of phase function behavior. Therefore, the OMC intends to experimentally approximate the shape factors of various basic shapes by imaging a suite of Lambertian objects with varying geometries. By isolating the shape factor as the only unknown term in the space illuminance ratio, it is possible to identify the impact of a target's shape on phase function, which will ultimately aide in the identification of unknown targets, in addition to improving the accuracy of phase function approximations utilized in subsequent size estimations.

While the effort of experimentally approximating the shape factors of basic geometries is a useful demonstration of the impact that a body's shape has on its photometric properties, the utility in applying these approximations directly to size estimations 

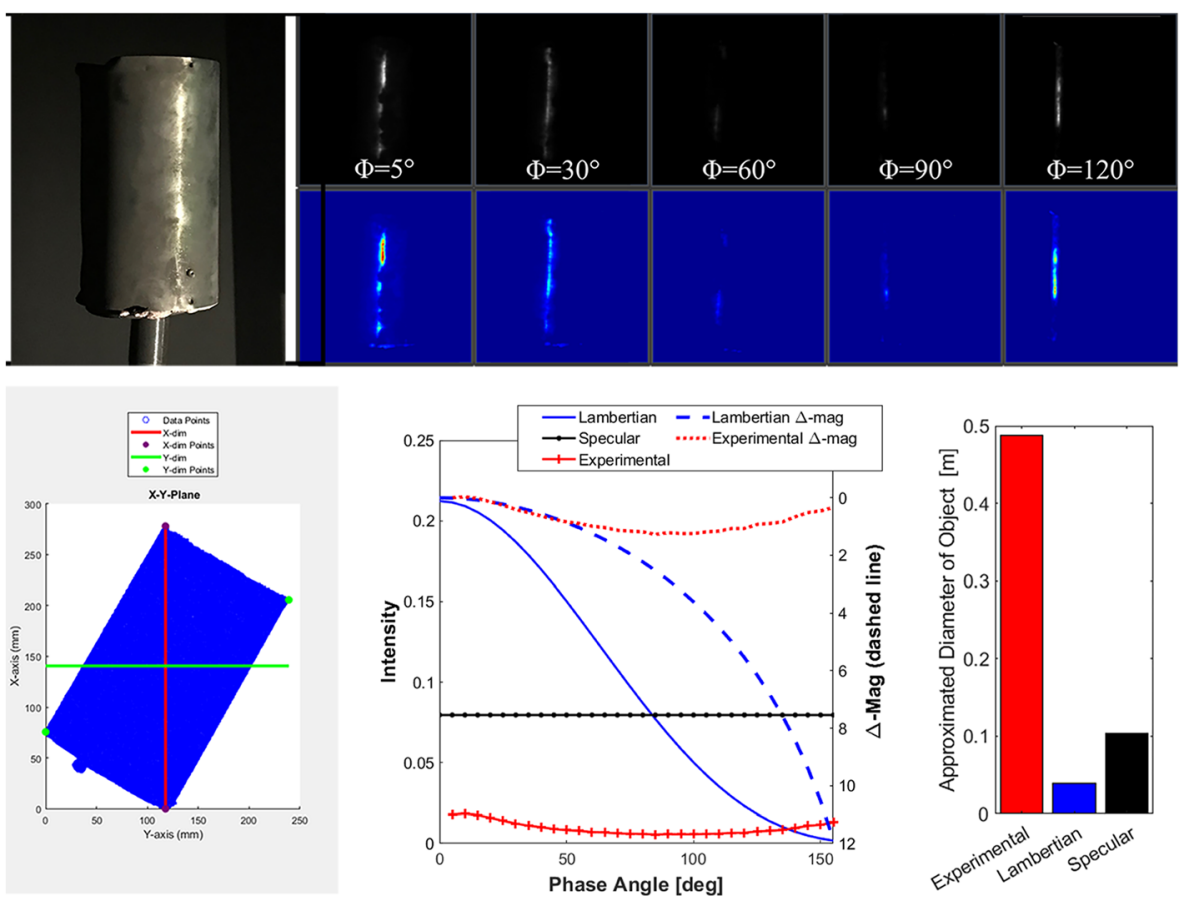

Fig. 9 Top: (left) telescope frame fragment from DebriSat as viewed from the CCD and (right) representative binary and color map renderings of images at 5-, 30-, 60-, 90- and 120-degree phase angles. Bottom: (left) point cloud analysis of X-Y plane of telescope frame used to calculate $\mathrm{L}_{\mathrm{c}}$, (center) experimental phase functions generated for the telescope frame in comparison to Lambertian and Specular phase functions, and (right) comparative size estimations for approximated diameter " $\mathrm{d}$ " (green dashed-line) as used in optical size estimation resulting from theoretical specular, theoretical Lambertian, and experimental Lambertian phase functions

made for unknown orbital debris is complicated. Orbital debris generated via impact, break-up, or any such kinetic mechanism is unlikely to result in bodies that have a simplistic geometric shape, as has been shown with the DebriSat fragments. Therefore, the OMC intends to utilize the multitude of DebriSat fragments, which are categorized into six shape categories. These fragments have been imaged to create precise point clouds used to calculate the $\mathrm{L}_{\mathrm{c}}$ among other parameters and to validate the laboratory experimentally derived-phase functions against 3D model ray-tracing software. The ray-tracing software will allow for rapid parametric analyses of target characteristics on

Table 3 Comparative Size Estimations for Telescope Frame

\begin{tabular}{|c|c|c|c|c|}
\hline & \multicolumn{2}{|c|}{$\alpha_{\mathrm{g}}=0.175$} & \multicolumn{2}{|c|}{$\alpha_{\mathrm{g}}=0.3042$} \\
\hline & $\mathrm{d}[\mathrm{m}]$ & $\%$ Error & $\mathrm{d}[\mathrm{m}]$ & $\%$ Error \\
\hline Lambertian & 0.0392 & 86.52 & 0.0253 & 91.30 \\
\hline Specular & 0.1045 & 64.05 & 0.0675 & 76.78 \\
\hline Experimental & 0.4919 & 69.20 & 0.2907 & 0.00 \\
\hline
\end{tabular}


subsequent behaviors exhibited in the phase function. While these analyses would initially be confined to the influence of material composition, the OMC intends to pursue a shape factor study in parallel by implementing a triangular meshing approach for object modeling. It is hoped that through utilizing this approach, it is possible to approximate BRDFs of objects that have increasingly complex shapes and thereby increase the accuracy of experimentally derived-phase functions for objects that are more characteristic of orbital debris.

\section{Spectral Measurements}

One of the variables is remote optical measurements albedo variations due to space weathering and/or other external events (i.e., collision or explosion) that can affect the optical size calculations. Provided one albedo value is used for optical size calculations, beyond studying phase function and BRDFs, the following provides analysis of the spectral data that was collected on a range of materials pre-laboratory hypervelocity impact of DebriSat [6]. The goal is to provide an overview of typical space craft materials used in the construction of a modern-day satellite to establish if there are groupings or taxonomy-based relationships that could be applied in material assessments using remote observations. Fig. 10 provides an example of a typical set-up in the OMC using an Analytical Spectral Device (ASD) field spectrometer with a range from 350-2500 nm and a resolving power of approximately 200 (corresponding to a spectral resolution of $10 \mathrm{~nm}$ at $2000 \mathrm{~nm}$ ), and 717 channels that serve to obtain baseline spectral data on various material types. The system only needs 210 channels to obtain the desired bandwidth/ spectral resolution, so using 717 channels is over-sampling. Measurements are acquired by placing the target under the quartz lamp illuminator and orienting the spectrometer's fiber feed (mounted in a pistol grip) approximately perpendicular to the target surface. Depending on the reflectance properties of the target/material, the angle of incidence and detector direction are modified to best sample the material with maximum signal and without saturating the system. The data are output to a laptop computer and reduced using in-house developed software to provide the absolute spectral reflectance. The processed data are uploaded to the NASA Johnson Space Center (JSC) Spacecraft Materials Spectral Database and available upon request and approval to U. S. citizens. A subset of spectral reflectance data on typical spacecraft materials used in DebriSat will be presented in Figs. $11,12,14$ and 15. All figures below are plotted as a function of absolute reflectance on the y-axis, where 1.0 would be a perfect diffuse reflector as calibrated against a Spectralon calibration plate, as a function of wavelength. For values that extend beyond 1.0, the reflectance may have saturated the detector as observed with highly specular material or components, illustrated in Figs. 12 and 15.

The Ultra Triple Junction (UTJ) solar cells used for constructing the DebriSat mockup satellite are composed of a GaInP2/GaAs/Ge structure above a germanium substrate and coated with anti-reflective cover glass for maximum absorption [20]. The reflectance spectrum of the DebriSat UTJ solar cell, shown in Fig. 11, was measured and compared to various additional solar cell samples of similar composition for optical data comparison. Germanium-based photovoltaic cells have consistently produced a reflectance spectrum with an apparent peak present between $850 \mathrm{~nm}$ to $900 \mathrm{~nm}$, a characteristic seen in all four data samples shown in the figure. The dual junction GaAs/Ge structured solar cell doped above a germanium substrate, and Spectrolab UTJ 


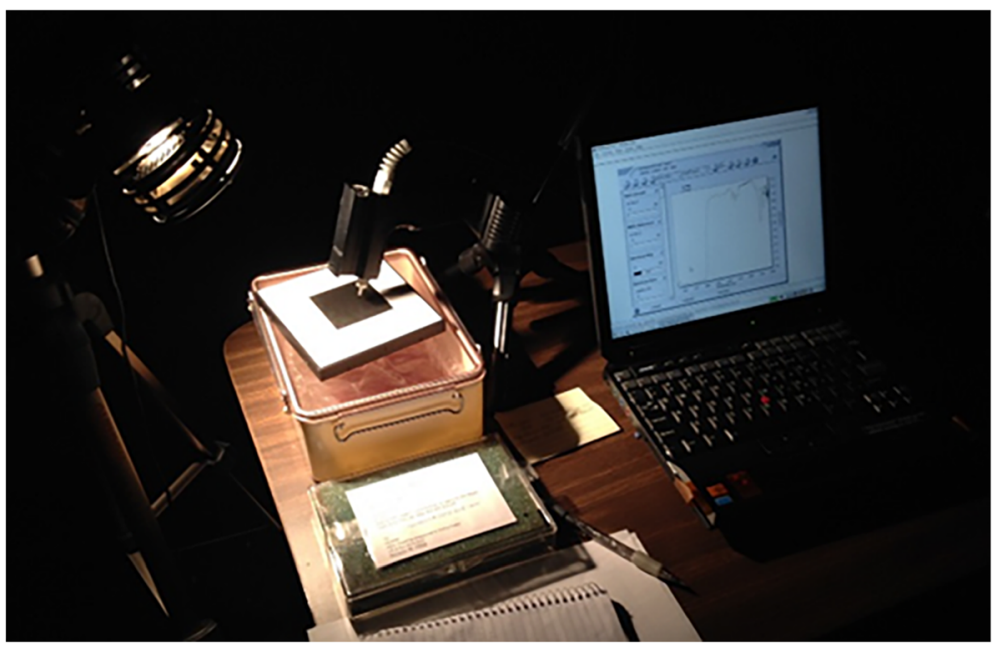

Fig. 10 ASD Spectrometer set-up with material placed on a Spectralon panel, quartz lamp, and pistol fiber feed to spectrometer/computer. The set-up shows an approximate 90-degree phase angle from illumination source-target-detector

and Improved Triple Junction (ITJ) solar cells all show a typical carbon-hydrogen (C$\mathrm{H})$ bond feature near $2300 \mathrm{~nm}$. It is not clear why the DebriSat UTJ solar cell does not show a strong indication of this feature.

Optical reflectance data of DebriSat Aluminum 6061 alloy samples coated with diverse colors are displayed in Fig. 12. Metals are optically opaque, making their distinguished pigmentation discernable by reflectance within the visible region of the spectrum [4]. This is possible because light impinging on the material surface will excite electrons to unoccupied sites of higher energy, resulting in the absorption and reflectance of different light colors [4]. Hence, the blue anodized Aluminum 6061

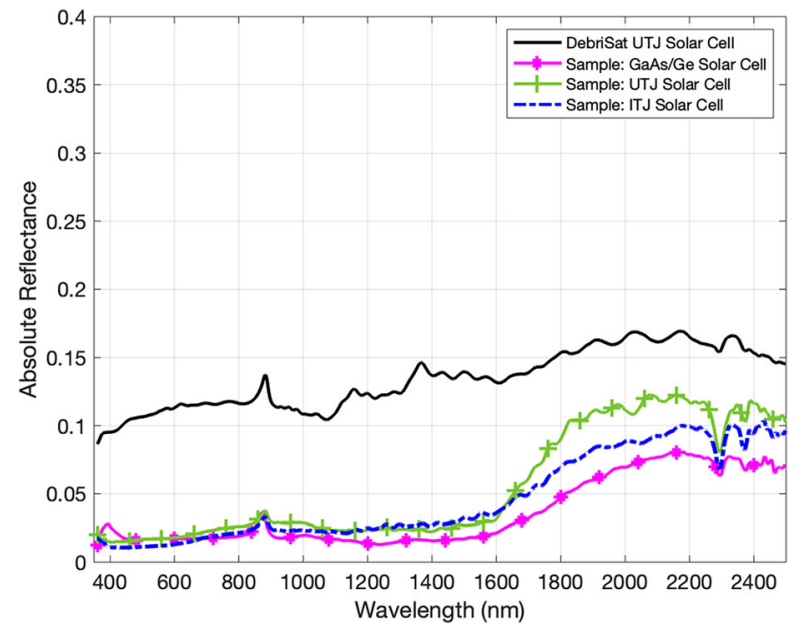

Fig. 11 Reflectance spectrum for DebriSat UTJ solar cell and various solar cell samples of similar material composition. Overall, the DebriSat solar cell had a higher spectral reflectance in comparison to other solar cells, but also did not exhibit a typical increase in reflectivity (slope) near $1600 \mathrm{~nm}$ 


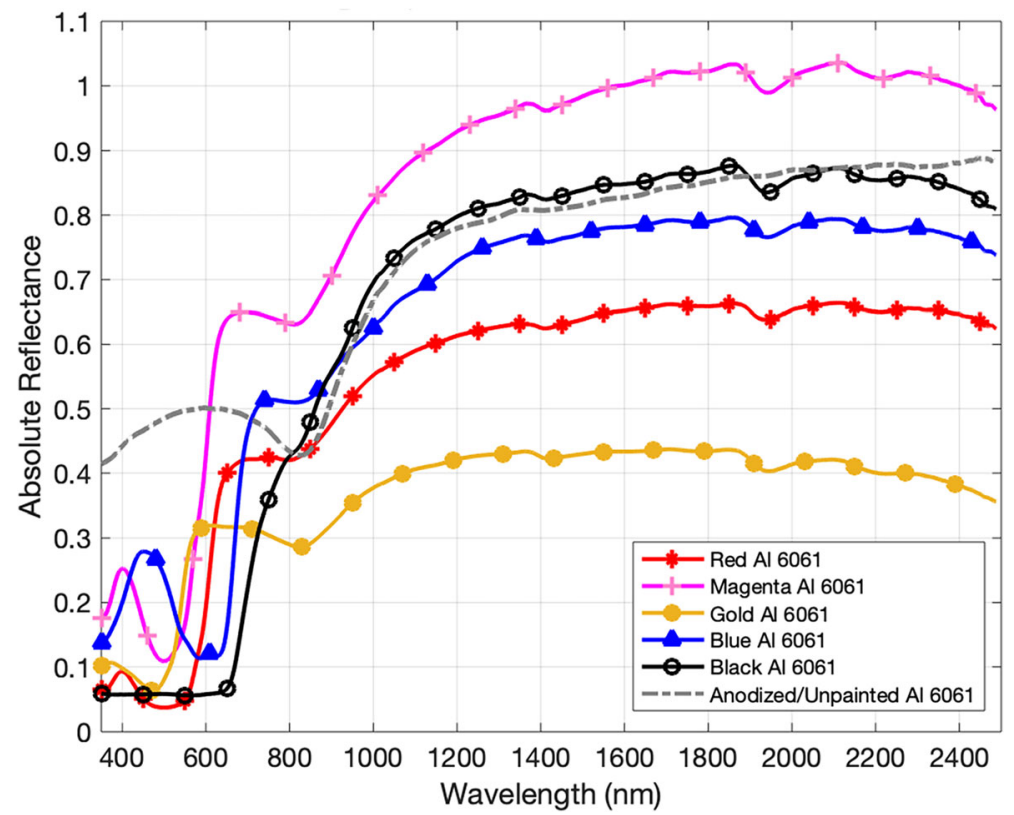

Fig. 12 Reflectance spectrum for DebriSat Aluminum 6061 (Al 6061) anodized in different colors (used for backtracking the location of the fragment to the pre-impacted satellite) in comparison to clear anodized $\mathrm{Al}$ 6061. The clear anodized/unpainted spectra had a higher reflectance than the colored anodized samples from $350 \mathrm{~nm}$ to approximately $600 \mathrm{~nm}$. For wavelengths outside the visible regime, magenta dominated the spectral, followed closely by black and clear anodized aluminum, followed by blue, red, and gold aluminum in that order

sample produced initial prominent reflectance at $\sim 400 \mathrm{~nm}$, whereas the gold- and redcolored alloy samples exhibit heightened reflectance near 550-600 nm (Fig. 12). The magenta-coated aluminum alloy sample resulted in a modest amount of reflectance at $\sim 380 \mathrm{~nm}$ coupled with substantial reflectance at $600 \mathrm{~nm}$, symbolizing purple and red color components in the magenta pigment. The black anodized Aluminum 6061 sample showed a featureless, low reflectance through the entire visible region $(350-700 \mathrm{~nm})$, typical for materials that are black. The clear anodized aluminum alloy produced a higher reflectance response between 350-700 nm, differentiating itself from all other anodized samples, due to the high reflectivity seen in bare metals [4]. A spectral feature common amongst all Aluminum 6061 alloy samples belongs to the absorption feature present at $850 \mathrm{~nm}$; an optical property typical of most aluminum metals [8]. When analyzing optical behavior of the measured alloy samples in the near infrared region beyond $1000 \mathrm{~nm}$, the colored-anodized aluminum samples produced common absorption features at $1400 \mathrm{~nm}$ and $1900 \mathrm{~nm}$, associated with water, and a wide absorption feature centered at $2200 \mathrm{~nm}$, indicating the presence of organic content likely resulting from carbon-hydrogen $(\mathrm{C}-\mathrm{H})$ bonds or a variation of oxygen-hydrogen $(\mathrm{X}-\mathrm{O}-\mathrm{H})$ bonds, an unknown element that bonds with $\mathrm{O}-\mathrm{H}[12,15]$. Therefore, the clear anodized alloy produced spectrum with no organic features and only the clear indication of aluminum. Using this data to differentiate between types of anodized Al 60601 may be best suited focusing on the visible and near-infrared regime and binning the reflectance spectra into broadband filters to produce color-color index groupings (i.e., B-R vs R-I). 
MLI (i.e., thermal blankets) components have been considered an essential material incorporated on the exterior of countless spacecraft articles. MLI functions as a substance employed to protect components, such as solid rocket motors and propellant tanks, from elevated temperatures due to external environmental sources while hindering the component from experiencing its own loss of heat [7]. A Kapton® polyimide film, manufactured by E.I. du Pont de Nemours and Co., was used in the DebriSat MLI. Trade names and trademarks are used in this report for identification only. Their usage does not constitute an official endorsement, either expressed or implied, by the National Aeronautics and Space Administration.

The DebriSat MLI Kapton polyimide shows optical similarities to that of two other polyimide samples studied previously (Fig. 13). An in-depth study of MLI components, such as the Space Facing Kapton and Spacecraft Facing Kapton data are provided in Rodriguez 2007. In an MLI blanket, the Spacecraft Facing Kapton sample resembles a woven quilt-like structure and it would be used closest to the spacecraft, while the Space Facing Kapton sample refers to the visibly external side of the polyimide sheet covering the spacecraft article and is perforated for thermal regulation [19]. The previously evaluated Space Facing and Spacecraft Facing polyimide data are compared alongside the DebriSat MLI Kapton polyimide for characteristic similarities. Coppercolored Kapton has a known absorption feature near $450 \mathrm{~nm}$, which is seen in all three data samples in Fig. 13. The DebriSat MLI that was measured resembled the space facing structure, but it is interesting to note that the overall reflectance for the DebriSat MLI polyimide sample remains at an intermediate value between Space Facing and Spacecraft Facing samples over the majority of the spectral response.

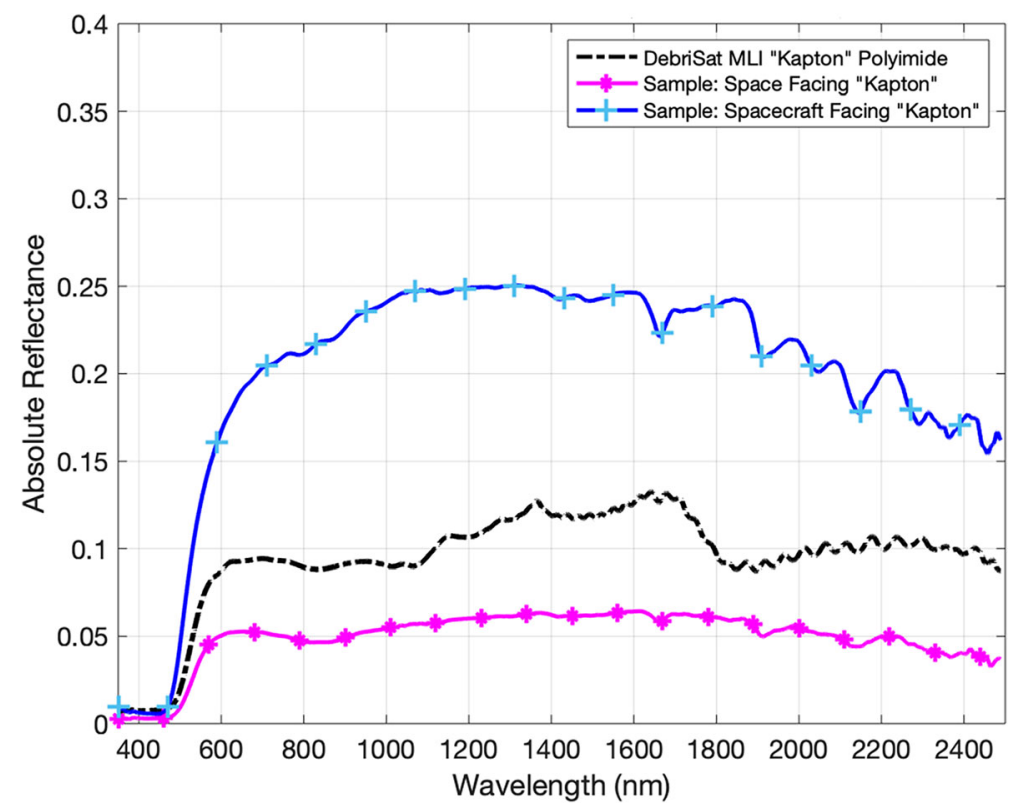

Fig. 13 Reflectance spectrum for DebriSat MLI wrap Kapton polyimide sample compared with two other Kapton polyimide copper-colored samples. The DebriSat sample has an overall higher reflectance throughout the spectral instrument bandpass and exhibits similar absorption features typical of copper-colored polyimide film 
A Composite Overwrapped Pressure Vessel (COPV) used in DebriSat and visual "gold shimmer" DebriSat composite materials were also compared with similar composite materials in terms of measured absolute spectral reflectance. The DebriSat COPV and gold shimmer composite materials had equal configurations consisting of an Aluminum-6061-T6 honeycombed core embedded within a Toray T1000 composite. Previous studies performed in the realm of material optical properties claim that composites made of carbon fiber produce reflectance independent of surface roughness, are Lambertian rather than specular, and are dependable absorbers comparable to material absorbers designated as good standards for calibration purposes [3]. This is likely the reason we see relatively low absolute reflectance signatures less than 0.45 produced by the DebriSat composites and sample composites within 350-2500 nm in Fig 14.

With a tensile strength of $924 \mathrm{ksi}$ and low density of $1.8 \mathrm{~g} / \mathrm{cm}^{3}$, Toray carbon fiber composites are commonly utilized in space, military, and commercial based applications [21, 22]. Therefore, it is essential to discern the optical signature produced by composites to aid in remote observations telescopically. The "DebriSat Composite: Gold Shimmer" appeared dominantly black in color, though had a gold shimmer tint to its surface upon light impinging on it. This gold shimmer was also present on composite samples 1, 2, and 3, plotted in Fig. 14 for comparison, but this was not the case for the black COPV DebriSat composite. Composites are well known to incorporate epoxy resin in their structure for enhanced physical properties and generate a yellow amber hue that is seen in resins having low-molecular-weight or medium viscosity $[1,23]$. In general composites, predominately black in color, share a

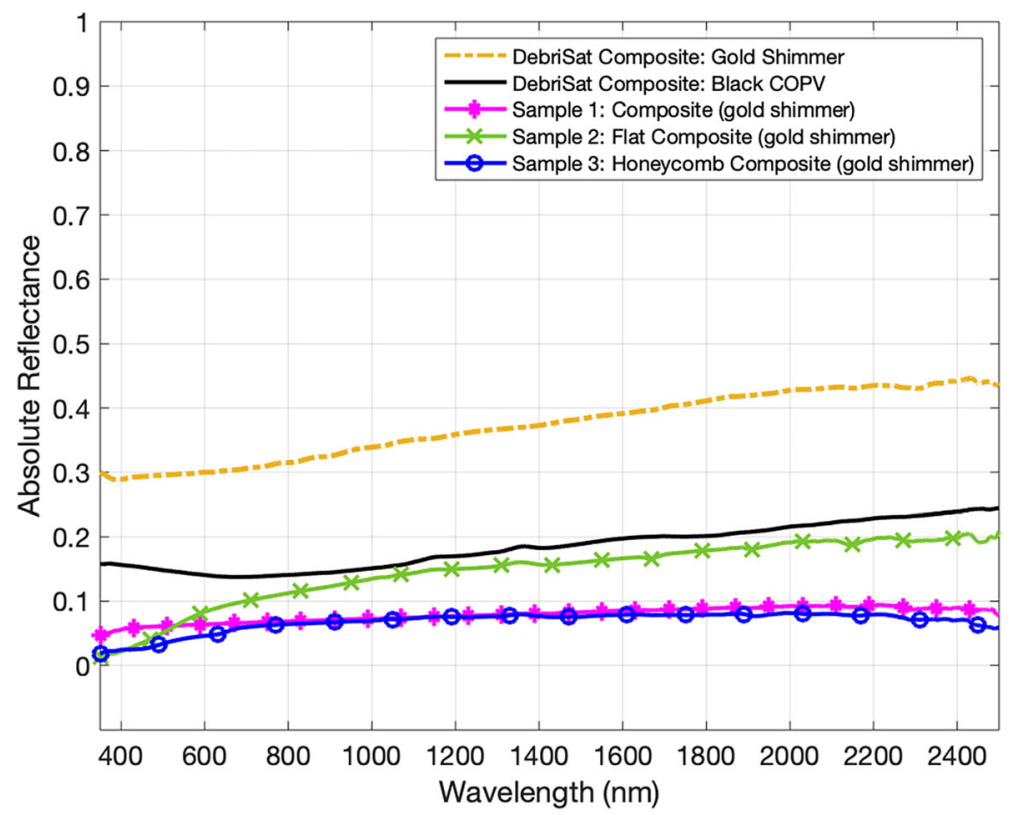

Fig. 14 Reflectance spectrum for a set of DebriSat composite material specimen compared with spectra belonging to previously measured spacecraft composite samples. The DebriSat samples (black and gold) were both more reflective over the 350-2500 $\mathrm{nm}$ than the comparative samples and showed an overall featureless data curve with a slight increase in slope approaching the near infrared regime 
featureless spectral signature, although the two DebriSat samples and the flat composite sample show a slight increasing trend through the infrared wavelengths.

Several printed circuit boards of different colors were incorporated into the DebriSat experiment and have had their optical reflectance properties measured. Between all seven circuit boards tested, a total of four different companies supplied the samples, therefore not all circuit boards were provided by the same manufacturer. Yet, similarities in spectral characteristics are identified in most of the circuit board samples, specifically the organic features in the infrared section beyond $800 \mathrm{~nm}$. There are common absorption bands in the different colored circuit boards observed at $1450 \mathrm{~nm}$, $\sim 1700 \mathrm{~nm}, 1900 \mathrm{~nm}$, and $2300 \mathrm{~nm}$ in Fig. 15. The absorption band present at 1700 $\mathrm{nm}$, and $2300 \mathrm{~nm}$ are likely due to $\mathrm{C}-\mathrm{H}$ bands, while the features at $\sim 1400 \mathrm{~nm}$ and $1900 \mathrm{~nm}$ suggest the typical $\mathrm{H}_{2} \mathrm{O}$ (water band) presence [15]. Regarding color spectrums, each colored circuit board will have a bandgap due to its respective color; blue has absorption features between $\sim 350 \mathrm{~nm}$ (below the signal of the ASD spectrometer) and $400 \mathrm{~nm}$, green between $450 \mathrm{~nm}$ and $550 \mathrm{~nm}$ and red near $600 \mathrm{~nm}$ to $650 \mathrm{~nm}$. Like black, brown also has a featureless, low reflectance, but shows a slight, increasing reflectance through the visible and near-infrared wavelengths.

\section{Summary and Path Forward}

This paper reviewed the current status of DebriSat, hypervelocity impact experiment on a representative of modern spacecraft in the LEO environment. The data provided the

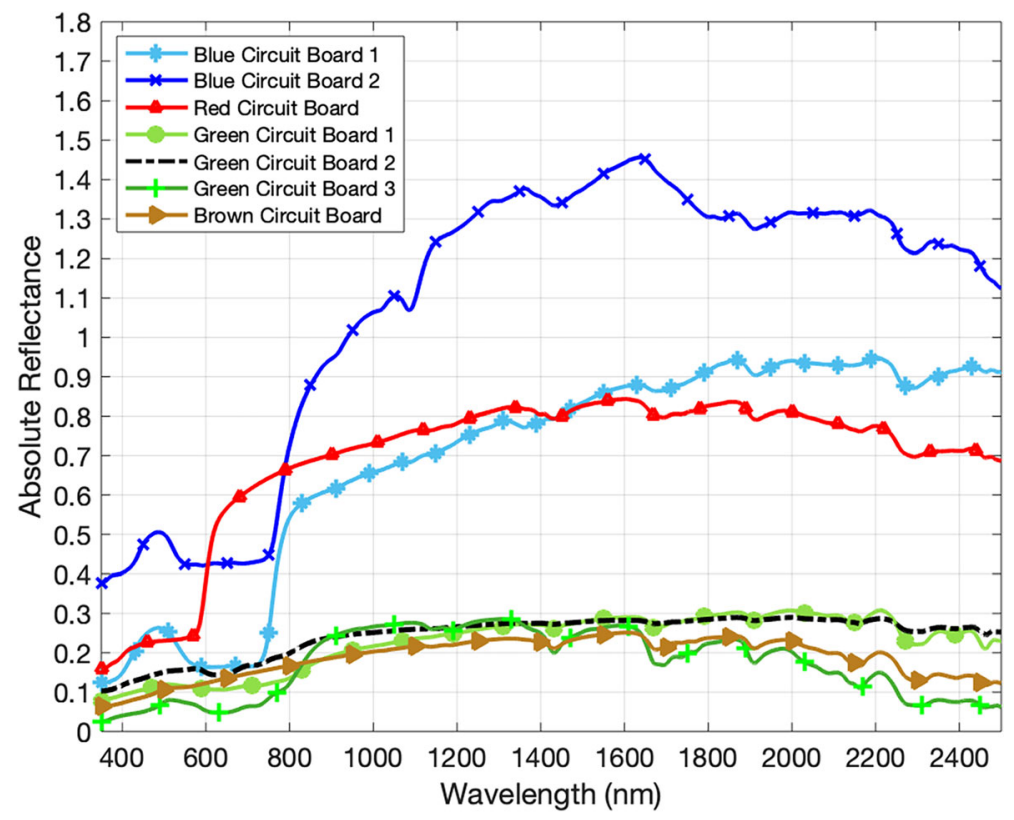

Fig. 15 Reflectance spectrum for multiple DebriSat circuit board components of various colors (blue, red, green, and brown). Circuit boards are nonhomogeneous materials in general, thus the presence, or lack thereof, spectral features may be a result of where the spectrometer field of view was placed on the target's surface. The variation in spectral plots are attributed to the solar of the circuit board measured 
results to date of the collected materials, sizes, and shapes that represent the fragmentation event.

Photometric-based phase functions and the best-fit for geometric albedo using a target of known size were presented with path forwards for the development of a more sophisticated phase function. These laboratory results would be used in parallel with optical telescopic data to improve the current optical SEM.

Characterizing the complexities using spectral measurements of spacecraft materials was also presented in finding potential groupings/taxonomies. Future research would incorporate color-color index data utilizing the same optical filters (i.e., bandpasses) as optical telescopes from the spectral data to determine if subsets of spacecraft materials could be isolated in this taxonomy research.

The work presented in this paper will be used not only to improve the optical SEM, but to improve the SSBM and incorporate shape parameterization into the next version of ORDEM.

Funding This work was funded by National Aeronautics and Space Administration.

Data Availability (https://amostech.com/TechnicalPapers/2020/Orbital-Debris/Cowardin.pdf)

Code Availability Not applicable.

\section{Declarations}

Conflict of Interest The authors declare no conflict of, nor competing interest in the creation of this work.

Open Access This article is licensed under a Creative Commons Attribution 4.0 International License, which permits use, sharing, adaptation, distribution and reproduction in any medium or format, as long as you give appropriate credit to the original author(s) and the source, provide a link to the Creative Commons licence, and indicate if changes were made. The images or other third party material in this article are included in the article's Creative Commons licence, unless indicated otherwise in a credit line to the material. If material is not included in the article's Creative Commons licence and your intended use is not permitted by statutory regulation or exceeds the permitted use, you will need to obtain permission directly from the copyright holder. To view a copy of this licence, visit http://creativecommons.org/licenses/by/4.0/.

\section{References}

1. Bank, L.C: Composites for Construction: Structural Design with FRP Materials. John Wiley \& Sons, Inc. (2006)

2. Barker, E., et al.: Analysis of Working Assumptions in the Determination of Populations and Size Distributions of Orbital Debris from Optical Measurements, Proceedings of the 2004 AMOS Conference, Maui Economic Development Board, Maui, Hawaii, 225-235 (2004)

3. Burchell, T.D.: Carbon Materials for Advanced Technologies, 1st edn. Pergamon, Amsterdam (1999)

4. Campbell, F.: Elements of Metallurgy and Engineering Alloys, Materials Park, OH, ASM International, 321-322 (2008)

5. Cowardin, H., et al.: Updates to the DebriSat project in support of improving breakup models and orbital debris risk assessments. ASME. (2019). https://doi.org/10.1115/HVIS2019-066

6. Fibre Glast Developments Corporation: System 2000 Epoxy Resin. Brookeville, Ohio http://cdn. fibreglast.com/downloads/00343-A.pdf (2020). Accessed 8 December 2020 
7. Gilmore, D., Donabedian, M.: Spacecraft Thermal Control Handbook: Fundamental Technologies, vol. 1, 2nd edn. The Aerospace Press (2002)

8. Hatch, J.: Aluminum - Properties and Physical Metallurgy. ASM International, Metals Park, Ohio (1984)

9. Hejduk, M., Cowardin, H., Stansbery, E.: Satellite Material Type and Phase Function Determination in Support of Orbital Debris Size Estimation, Proceedings of the 2012 AMOS Conference. https://amostech. com/TechnicalPapers/2012/Orbital_Debris/HEJDUK.pdf (2012). Accessed 8 December 2020

10. Hostetler, J., Cowardin, H.: Experimentally-Derived Bidirectional Reflectance Distribution Function Data in Support of the Orbital Debris Program Office, Proceedings of the 2019 AMOS Conference. https://amostech.com/TechnicalPapers/2019/Orbital-Debris/Hostetler.pdf (2019a). Accessed 8 December 2020

11. Hostetler, J., Cowardin, H.: Experimentally-Derived Phase Function Approximations in Support of the Orbital Debris Program Office, First International Orbital Debris Conference, Houston, Texas' https:// www.hou.usra.edu/meetings/orbitaldebris2019/orbital2019paper/pdf/6148.pdf (2019b). Accessed 8 December 2020

12. Jorgensen, K.: Using Reflectance Spectroscopy to Determine Material Type of Orbital Debris, ProQuest Dissertations And Theses; Thesis (Ph.D.)-University of Colorado at Boulder, 2000.; Publication Number: AAI9969379; ISBN: 9780599742468; Source: Dissertation Abstracts International, Volume: 61-04, Section: B, page: 2054.; 189 p. (2000). https://ui.adsabs.harvard.edu/abs/2000PhDT.......43J/ abstract

13. Liou, J.-C.: An Update on Recent Major Breakup Fragments, Orbital Debris Quarterly News 13(3), 5-6. https://orbitaldebris.jsc.nasa.gov/quarterly-news/pdfs/odqnv13i3.pdf (2009). Accessed 8 December 2020

14. Liou, J.-C., et.al.: Successful Hypervelocity Impacts of DebrisLV and DebriSat, Orbital Debris Quarterly News18(3), 3-6, https://orbitaldebris.jsc.nasa.gov/quarterly-news/pdfs/odqnv18i3.pdf (2014). Accessed 8 December 2020

15. Mao, J., Bierlein, F.: Mineral Deposit Research: Meeting the Global Challenge, vol. 1. Springer-Verlag (2005). https://doi.org/10.1007/3-540-27946-6

16. Mulrooney, M., et al.: An Investigation of Global Albedo Values, Proceedings of the 2008 AMOS Conference. https://amostech.com/TechnicalPapers/2008/Posters/Mulrooney.pdf (2008). Accessed 8 December 2020

17. Murray, J., et al.: Analysis of the DebriSat Fragments and Comparison to the NASA Standard Satellite Breakup Model, First International Orbital Debris Conference, Houston, Texas https://www.hou.usra. edu/meetings/orbitaldebris2019/orbital2019paper/pdf/6135.pdf (2019). Accessed 8 December 2020

18. Nicodemus, F., et al.: Geometrical Considerations and Nomenclature for Reflectance. United States. National Bureau of Standards, U.S. Govt. Print. Off. https://www.worldcat.org/title/geometricalconsiderations-and-nomenclature-for-reflectance/oclc/3121126\&referer=brief_results (1977). Accessed 8 December 2020

19. Rodriguez, H., Abercromby, K., Mulrooney, M., Barker, E.: Optical Properties of Multi-Layered Insulation, Proceedings of the 2007 AMOS Conference. https://amostech.com/TechnicalPapers/2007/ Poster/Rodriguez.pdf (2007). Accessed 8 December 2020

20. Spectrolab Inc.: Ultra Triple Junction (UTJ) Data Sheet, Sylmar, CA https://www.spectrolab.com/ photovoltaics/UTJ-CIC_Data_Sheet.pdf (2008). Accessed 8 December 2020

21. Toray Composite Materials America, Inc.: Aerospace Prepreg Selector Guide https://www.toraycma. com/files/library/fd863a2f973d87c0.pdf (2017). Accessed 8 December 2020

22. Toray Composite Materials America, Inc.: T1000G Intermediate Modulus Carbon Fiber https://www. toraycma.com/file_viewer.php?id=5851 (2018). Accessed 8 December 2020

23. Wang, R., Zheng, S., Zheng, Y.: Polymer Matrix Composites and Technology, Cambridge, Woodhead Publishing, eBook ISBN: 9780857092229 (2011)

Publisher's Note Springer Nature remains neutral with regard to jurisdictional claims in published maps and institutional affiliations. 